\title{
Avulsion Fracture of the Gracilis in an Adolescent Swimmer
}

\author{
Britnie Jones ${ }^{1}$ and Dani Moffit ${ }^{2 *}$ \\ ${ }^{1}$ University of Oregon, USA \\ ${ }^{2}$ Program Director - Master of Science in Athletic Training, USA
}

*Corresponding author: Dani Moffit, Associate Professor, Program Director - Master of Science in Athletic Training, 912 S 8th Ave, Stop 8105, Pocatello, ID 83209-8105, USA

Submission: 畊January 19, 2018; Published: 侮 February 08, 2018

\begin{abstract}
Objective: To describe evaluation and treatment for an avulsion fracture in a sport where this injury is rare.

Background: Adolescents present with the highest number of avulsion fractures due to late ossification between the apophysis and body of the bone causing a weakness in the transfer of force between muscle and bone. This injury is highest in soccer players and gymnasts, specifically at the ischial tuberosity, anterior inferior iliac spine, and anterior superior iliac spine. Gracilis avulsion fractures are rare in swimmers when compared to other sports.
\end{abstract}

Differential Diagnosis: Groin strain, sports hernia, osteitis pubis.

Treatment: After diagnosis was confirmed through diagnostic ultrasound, a 16-week protocol was followed, similar to one used for hernia repairs, for rehabilitation purposes.

Uniqueness: The original mechanism of injury was caused by internal hip rotation on a planted pivot foot, uncommon for groin strains. The most common mechanism of injury for the secondary avulsion injury is eccentric overload, however this was not the case for this swimmer. The gracilis avulsion occurred while non-weight bearing in a pool. His body weight, being affected by buoyancy, should have reduced the amount of tension on the muscles by approximately 50 percent. Therefore, his concentric muscle action should not have produced the amount of force necessary to avulse the gracilis.

Conclusion: A paucity of literature indicates gracilis avulsion fractures are rare in swimmers. Both conditions in this athlete reported unique mechanisms of injury for sustaining a groin strain or avulsion. Athletic trainers should be aware of the potential for avulsion fractures regardless of the sport or mechanism of injury reported.

Keywords: Avulsion fracture; Swimming; Gracilis apophysitis; Pubic avulsion

\section{Introduction}

Avulsion fractures of the apophyses are diagnosed with high frequency in adolescent athletes as a result of eccentric muscle contraction or a sudden unbalanced contraction of an attached musculotendinous unit [1,2]. The ischial tuberosity, anterior inferior iliac spine, anterior superior iliac spine, and superior corner of the pubic symphysis are the most common sights for avulsion fractures in adolescent athletes [1-3]. Athletes competing in soccer and gymnastics present the highest number of avulsion fractures, whereas swimmers are more likely to suffer injuries at the shoulder, back, and knee $[1,2,4]$. Gracilis avulsion fractures in swimmers are rarely reported in literature.

\section{Case Report}

A 15-year old male interscholastic breaststroke-specialty swimmer with no prior history of groin pathology was participating in dry land training when he reported feeling a pop in his right groin. The mechanism of injury was an internal rotation of his right hip during a pivot motion. Initially treated as an adductor strain, the patient returned to physical activity the next day, only complaining of groin pain. Seven days later while pushing off the bottom of the pool, he performed a motion similar to the breaststroke kick. During adduction with his umbilicus approximately 2 inches below water level, he felt a pop in his right groin. He experienced immediate pain followed by ecchymosis in his right upper groin near the inguinal crease.

The athlete was taken to the physician for evaluation. Ultrasound revealed a normal adductor longus, brevis, and magnus. The gracilis showed a hyperechoic area in the proximal muscle and an avulsion of the apophyseal attachment (Figure 1). The physician diagnosed right adductor tendonitis/gracilis apophysitis. The physician's initial assessment described the athletes' right iliac crest and posterior superior iliac spine (PSIS) as being significantly 
higher than the left, a dysfunctional right sacroiliac joint, and limited hip, quadriceps, and hamstring flexibility. The patient was referred for strengthening and flexibility rehabilitation for six weeks. Treatment included light stretching of adductors, piriformis, hamstrings and IT band, with joint soft tissue mobilizations of the pelvis and surrounding soft tissue. Modalities included ultrasound administered near the avulsion at $1 \mathrm{MHz} 0.8 \mathrm{~W} / \mathrm{cm}^{2}$ for 8 minutes and IFC stim with ice for $15 \mathrm{~min}$ throughout the duration of therapy.

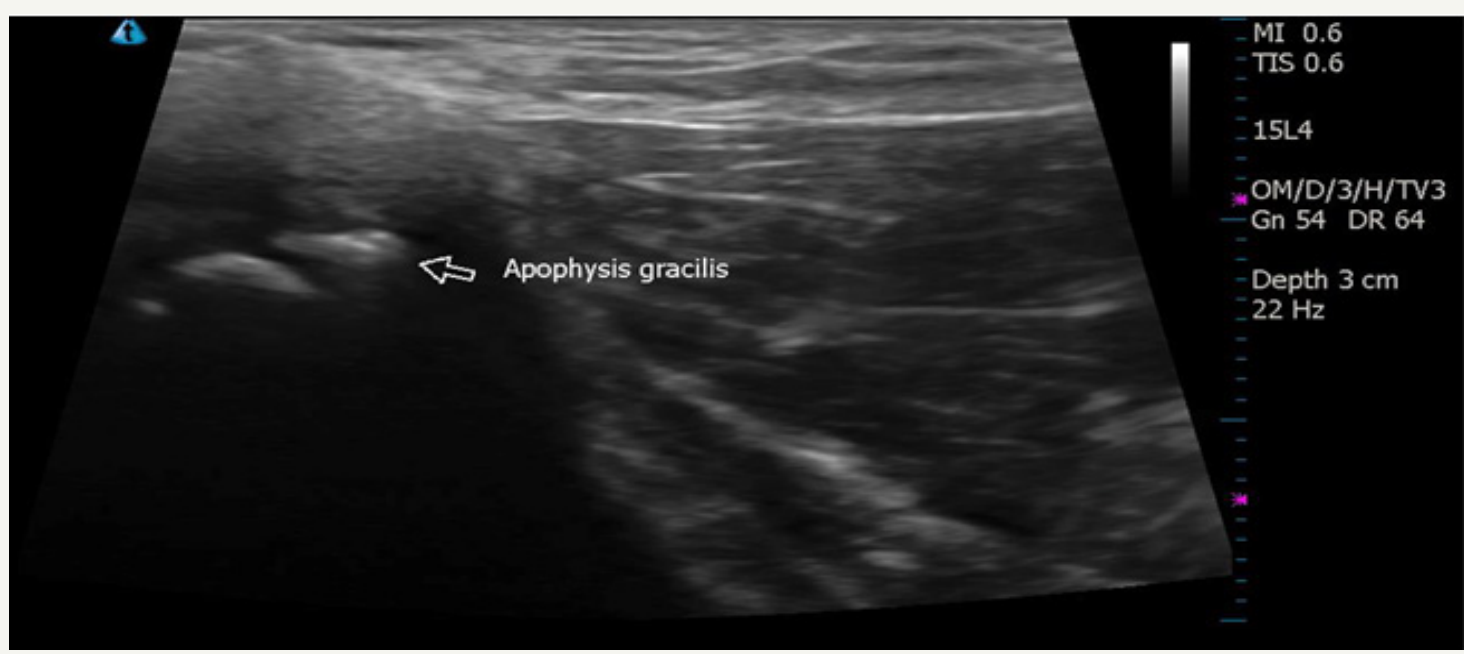

Figure 1: Diagnostic ultrasound image reveals hyperechoic area distal to the apophysis of the gracilis and an avulsion of the gracilis (arrow).

Throughout rehabilitation the patients' swimming activity levels varied. During week one of therapy the patient was not allowed to participate in swimming. He then progressed to upper body pulling (no kick) at two weeks post-injury. At week four he was able to include light kicking (flutter or dolphin). Weeks five and six he returned to full swimming but was self-limiting due to pain. The patient was discharged from therapy after 16 visits. He returned to full workout status with complaints of pain during breaststroke kicks only.

Five months after the initial injury, the patient began complaining of right groin pain. Following a swimming practice session, the patient's right leg appeared to be swollen. A physician's examination alluded to deep vein thrombosis (DVT), so he was referred for an ultrasound. The technician did not find a DVT, but thought there may be an inguinal hernia. The patient returned to the original physician who diagnosed the apophysitis. Diagnostic ultrasound imagining showed right femoral acetabular impingement and upper rectus femoris tendonitis.

The physician placed him on a hernia rehabilitation protocol, which he completed with the athletic trainer during weight sessions at practice. The protocol included abdominal strengthening (e.g., planks, leg lifts), hip and pelvis exercises (e.g., bridging, bird dogs), and adductor strengthening (e.g., clams shells, lunges with theraband). The exercises were followed for six weeks before he was released to continue weights with the team.

\section{Discussion}

The most common injury sites for Division I collegiate swimmers include the shoulder, neck, back, and knees [4,5]. Most injuries occurred in freshmen swimmers when compared to other class levels; the age most similar to our patient [4,5]. A study of 296 breaststroke swimmers found that $42.7 \%$ of subjects were unable to compete due to hip adductor injuries, though none were reported as avulsion fractures [6]. Hip adductor pathologies may be seen in breaststroke swimmers due to adduction velocities reaching up to $245^{\circ}$ per second, muscular tightness, and the increased number of miles breaststrokers swim on average [6].

The initial mechanism of injury most likely placed him at risk for the injury in the pool. The mechanism of injury in the pool, coupled with his body immersion depth brings into question the contribution buoyancy to the etiology of this injury. His body weight should have been reduced by approximately 50 percent therefore reducing the amount of muscular strain [7]. As stated previously, velocities during hip adduction can reach up to $245^{\circ}$ per second, however we believe our patient was not strong enough to produce such force, bringing us to question the effects of hip alignment abnormalities. Our patient's right iliac crest and PSIS were significantly higher than the left, he had a dysfunctional right sacroiliac joint, with limited hip, quadriceps, and hamstring flexibility which are all potential factors that could cause a mechanical abnormality therefore increasing muscular tension while adducting his legs. Additionally, the femoral acetabular impingement may have contributed to the pain he was experiencing throughout the injury timeline.

\section{Conclusion}

The main objective of this case report is to increase the awareness of adductor avulsions in swimmers and the potential for other hip pathologies throughout recovery. Our patient continued to swim through groin discomfort throughout his return to competition, which may have affected his stroke pattern resulting in his second injury. Our case presents a unique mechanisms of injury for avulsion fractures, including: (a) concentric versus 
eccentric overload, (b) factors related to buoyancy, and (c) pelvic torsion contribution. These factors, potentially contributing to an adductor strain in an adult, manifested itself as an avulsion in an adolescent [8].

\section{Acknowledgement}

We would like to thank Dr. Tony Joseph for educating me on the diagnostic ultrasound images provided for this case.

\section{References}

1. Rossi F, Dragoni S (2001) Acute avulsion fractures of the pelvis in adolescent competitive athletes: prevalence, location and sport distribution of 203 cases collected. Skeletal Radiol 30(3): 127-131.

2. Porr J, Lucaciu C, Birkett S (2011) Avulsion fractures of the pelvis- a qualitative systematic review of the literature. J Can Chiropr Assoc 55(4): 247-255.

Creative Commons Attribution 4.0

International License

For possible submission use the below is the URL
3. Carl R (2012) Apophysitis and apophyseal avulsion of the pelvis. Int J Athl Ther Train 17(2): 5-9.

4. Chase K, Caine D, Goodwin B, Whitehead J, Romanick M (2013) A prospective study of injury affecting competitive collegiate swimmers. Res Sports Med 21(2): 111-123.

5. Wolf B, Ebinger A, Lawler M, Britton C (2009) Injury patterns in division I collegiate swimming. Am J Sport Med 37(10): 2037-2042.

6. Grote K, Lincoln T, Gamble J (2004) Hip adductor injury in competitive swimmers. Am J Sport Med 32(1): 104-108.

7. Cole AJ, Becker B (2004) Biophysiologic Aspects of Hydrotherapy. In: Comprehensive Aquatic Therapy. ( $2^{\text {nd }}$ edn), Elsevier Inc, Philadelphia, USA, pp. 19-56.

8. Anderson K, Strickland S, Warren R (2001) Hip and Groin Injuries in Athletes. Am J Sport Med 29(4): 521-533.

\section{Your subsequent submission with Crimson Publishers will attain the below benefits}

- High-level peer review and editorial services

- Freely accessible online immediately upon publication

- Authors retain the copyright to their work

- Licensing it under a Creative Commons license

- Visibility through different online platforms

- Global attainment for your research

- Article availability in different formats (Pdf, E-pub, Full Text)

- Endless customer service

- Reasonable Membership services

- Reprints availability upon request

- One step article tracking system 